\title{
Bavachalcone Enhances ROR $\alpha$ Expression, Controls Bmal1 Circadian Transcription, and Depresses Cellular Senescence in Human Endothelial Cells
}

\author{
Yanqi Dang, Shuang Ling, Jing Ma, Rongzhen Ni, and Jin-Wen Xu \\ Murad Research Institute for Modernized Chinese Medicine, Shanghai University of Traditional Chinese Medicine, \\ Shanghai 201203, China \\ Correspondence should be addressed to Jin-Wen Xu; jinwen.xu88@gmail.com
}

Received 26 February 2015; Revised 25 May 2015; Accepted 26 May 2015

Academic Editor: Jae Youl Cho

Copyright ( 2015 Yanqi Dang et al. This is an open access article distributed under the Creative Commons Attribution License, which permits unrestricted use, distribution, and reproduction in any medium, provided the original work is properly cited.

\begin{abstract}
The circadian clock regulates many aspects of (patho)physiology in the central nervous system and in the peripheral tissues. RAR-related orphan receptor $\alpha(\operatorname{ROR} \alpha)$, an orphan nuclear receptor, is involved in circadian rhythm regulation, including regulation of cardiovascular function. Bavachalcone, a prenylchalcone, is a major bioactive chalcone isolated from Psoralea corylifolia. This natural ingredient activated ROR $\alpha 1$ luciferase reporter activity on drug screening. In addition, bavachalcone induced ROR $\alpha 1$ expression in mRNA and protein levels in a dose-dependent manner and enhanced the circadian amplitude of Bmall mRNA expression after serum shock. Moreover, bavachalcone suppressed senescence in human endothelial cells and mRNA expression of $\mathrm{p} 16^{\text {ink4a }}$ (a marker of replicative senescence) and IL-1 $\alpha$ (a proinflammatory cytokine of the senescence-associated secretory phenotype). These inhibitory effects were partially reversed by the ROR $\alpha$ inhibitor VPR-66. Our results demonstrate that bavachalcone, as a natural medicine ingredient, has a pharmacological function in regulating ROR $\alpha 1$.
\end{abstract}

\section{Introduction}

The circadian clock not only controls the central nervous system, but also regulates many aspects of (patho)physiology in the peripheral tissues, including cardiovascular function. ROR $\alpha$, an orphan nuclear receptor, is involved in the circadian rhythm regulation. ROR $\alpha 1$ and $\operatorname{ROR} \alpha 4$, not $\mathrm{ROR} \alpha 2$ and ROR $\alpha 3$, are the predominant forms of ROR $\alpha$ expression in vascular endothelial cells, smooth muscle cells, and fibroblasts $[1,2]$. ROR $\alpha$ directly activates transcription of the circadian gene Bmall through conserved ROR $\alpha$ response elements [3, 4]. Homozygous Rora ( $\mathrm{sg} / \mathrm{sg}$ ) mutant mice exhibited an enhanced susceptibility to atherosclerosis and hypoalphalipoproteinemia [5]. In addition, ROR $\alpha$ has been identified as a regulator of human apo A-1, A-5, and C-3 gene expression [5-7]. ROR $\alpha$ not only suppressed TNF- $\alpha$-induced VCAM-1 and ICAM-1 expression in human endothelial cells [8], but also reduced oxidative stress through the induction of SOD2 and GPx1 expression [9]. Moreover, reduced circadian clock expression was closely associated with age in genetic hypertensive rats [10].

Bavachalcone, a prenylchalcone, is a major bioactive chalcone isolated from Psoralea corylifolia; it exhibits many biological activities. For example, Lee et al. reported dosedependent reduction of inducible nitric oxide synthase activity in activated microglial cells treated with bavachalcone [11]. In osteoclasts treated with bavachalcone, Park et al. observed reduced activation of MEK, ERK, and Akt and suppression of osteoclast differentiation through NFATc1 [12]. In addition, a strong inhibitory effect of bavachalcone against 2 important isoforms of UDP-glucuronosyltransferases (UGT), UGT1A1, and UGT1A7, was observed [13]. In a previous study, we found that bavachalcone activates AMPK kinase activity, promotes the expression of MnSOD, and reduces mitochondrial superoxide anion [14]. On drug screening, bavachalcone enhances ROR $\alpha 1$ expression. Therefore, this study investigated the pharmacological effects of bavachalcone on ROR $\alpha 1$. 


\section{Materials and Methods}

2.1. Cell Cultures. Human umbilical vein endothelial cells (HUVECs) (CRL-1730) were ordered from ATCC (Manassas, USA), and human embryonic kidney 293 (HEK-293) cells were purchased from the Institute of the Chinese Academy of Medical Sciences Cell Bank (Shanghai, China). HUVECs were maintained in a $37^{\circ} \mathrm{C}$ incubator supplemented with $5 \% \mathrm{CO}_{2}$ in Dulbecco's modified Eagle's medium (DMEM) containing $10 \%$ fetal bovine serum. The medium was changed every 2 days and the cells were passaged with trypsin-EDTA. The population-doubling level (PDL) was calculated after each passage as $\mathrm{PDL}=(\log 10 Y-\log 10 X) / \log 10^{2}$, where $Y$ is the number of cells counted at the end of the passage and $X$ is the number of cells seeded. Cumulative population doubling was calculated as the sum of all PDL the changes [15].

2.2. Serum Shock Procedures. Serum shock was performed as follows: approximately $1 \times 10^{6}$ cells per $60-\mathrm{mm}$ petri dish were plated 1 day prior to the experiment so that the cells would be approximately $80 \%$ confluent on the day of the experiment. The medium was exchanged with $0.3 \%$ serum DMEM medium for $16 \mathrm{~h}$. At time $t=0$, the medium was exchanged with serum-rich DMEM containing 50\% new born bovine serum with or without $20 \mu \mathrm{mol} / \mathrm{L}$ bavachalcone; after $2 \mathrm{~h}$, this medium was replaced with $0.3 \%$ serum DMEM with or without $20 \mu \mathrm{mol} / \mathrm{L}$ bavachalcone. At the indicated times, the petri dishes were washed twice with ice-cold phosphate buffered saline (PBS) and frozen on a liquid nitrogen layer or stored at $-70^{\circ} \mathrm{C}$ until the extraction of whole cell RNA.

2.3. ROR $\alpha 1$ Reporter Luciferase Plasmid Construct and Assay. The thymidine kinase $(-83$ to +91$)$ plus $3 \times$ RORE [TCG ACT CGT ATA ACT AGG TCA AGC GCT G] sequence was generated by inserting the corresponding annealed oligonucleotides into the Luc pGL3-basic plasmid. HEK293 cells were cultured in 24-well plates at a density of $2 \times 10^{5}$ cells/well. When the cell density reached $70 \%-80 \%$, the cells were used for transfection. In each well, $0.8 \mu \mathrm{g}$ of a reporter vector of the pGL3 $\mathrm{ROR} \alpha$ reporter firefly luciferase gene and $0.016 \mu \mathrm{g}$ of a reporter pRL-SV40 plasmid of the Renilla luciferase gene as normalization control were transfected and diluted with the FuGENE HD transfection reagent in the Opti-MEM transfection medium. The medium was replaced with normal DMEM $6 \mathrm{~h}$ after transfection. After incubation with bavachalcone (Shanghai Yuanye Bio-Technology Co., Ltd., Shanghai, China) for $16 \mathrm{~h}$, the activity of the luciferase reporter gene was assayed using the dual-luciferase reporter 1000 assay system and detected using a Varioskan Flash microplate spectrophotometer (Thermo Scientific, USA).

2.4. Quantitative Real-Time Polymerase Chain Reaction. Total RNA was extracted using TRIzol (Life Technologies, Carlsbad, USA) according to the manufacturer's instructions. Real-time polymerase chain reaction (PCR) amplification and detection were performed using the SYBR Green qPCR SuperMix-UDG with ROX (Life Technologies) in a fluorescence thermal cycler (StepOne real-time PCR system, Life Technologies) according to the manufacturer's protocol. Gene expression was normalized using GAPDH as a reference gene. The primers used in our study are as follows: $\operatorname{ROR} \alpha$, forward: $5^{\prime}$-CAGGCTTCTTTCCCTACTGTTCGT-3', reverse: $5^{\prime}$-CCGCTGCTTGTTTTGATAGTTCTC-3'; BMAL1, forward: $5^{\prime}$-CAATCCATACACAGAAGCAAACTAC-3', reverse: 5' -ACATCCTACGACAAACAAAAATCC-3'; P16-INK4A (CDKN2A), forward: $5^{\prime}$ TTTTCACTGTGTTGGAGTTTTCTGG-3', reverse: $5^{\prime}$ TGAGCTTTGGTTCTGCCATTTG-3'; IL-1 $\alpha$, forward: $5^{\prime}$ GCCCAAGATGAAGACCAACCAGT-3 ${ }^{\prime}$, reverse $5^{\prime}$-CCGTGAGTTTCCCAGAAGAAGAGG-3'; GAPDH, forward: 5'-CGCTGAGTACGTCGTGGAGTC-3', reverse: 5'-GCTGATGATCTTGAGGCTGTTGTC-3'; $18 \mathrm{~S}$ rRNA, forward: 5' -AGGTCTGTGATGCCCTTAGATGTC-3', reverse: $5^{\prime}$ TCCTCGTTCATGGGGAATAATT- $3^{\prime}$.

2.5. Western Blot. After treatment, the cells were centrifuged and lysed in Triton/NP-40 lysis buffer containing $0.5 \%$ Triton X-100, 0.5\% Nonidet P-40, $10 \mathrm{mmol} / \mathrm{L}$ Tris $(\mathrm{pH} 7.5)$, $2.5 \mathrm{mmol} / \mathrm{L} \mathrm{KCL}, 150 \mathrm{mmol} / \mathrm{L} \mathrm{NaCl}, 20 \mathrm{mmol} / \mathrm{L} \beta$-glycerolphosphate, $50 \mathrm{mmol} / \mathrm{L} \mathrm{NaF}$, and $1 \mathrm{mmol} / \mathrm{L} \mathrm{Na}_{3} \mathrm{VO}_{4}$, sonicated by JY92-2D ultrasonic homogenizer (NingBo Scientz Biotechnology Co., Ltd, Zhejiang, China) and centrifuged for $10 \mathrm{~min}$ at $10000 \mathrm{~g}$. The supernatant was employed for protein concentration measurement using a protein assay kit (BioRad, Hercules, CA, USA), and $30 \mu \mathrm{g}$ of protein was separated through sodium dodecyl sulfate-polyacrylamide gel electrophoresis and transfered to nitrocellulose membranes (Pall China, Shanghai, China). The membranes were blocked overnight with $5 \%$ nonfat dried milk in a buffer containing $140 \mathrm{mmol} / \mathrm{L} \mathrm{NaCl}, 20 \mathrm{mmol} / \mathrm{L}$ Tris-HCl (pH 7.5), and $0.1 \%$ Tween 20 and incubated with the following primary antibodies: ROR $\alpha$ rabbit polyclonal antibody (ab60134, Abcam, USA) and anti-GAPDH monoclonal mouse antibody (KangChen Bio-tech Inc., Shanghai, China). Finally, the membranes were incubated with a horseradish peroxidase (HRP) conjugated secondary antibody, at $4^{\circ} \mathrm{C}$ with gentle shaking overnight. The membranes were exposed to high-performance autoradiography film (Fuji film Co., Tokyo, Japan) and visualized using the ECL immobilon western chemiluminescent HRP substrate (WBKLS0500, Millipore, USA). Quantitative analysis was performed using Quantity One software (Bio-Rad Laboratories). Western blot experiments were performed in triplicate.

2.6. $\beta$-Galactosidase Senescence Staining. For antisenescence experiments, human endothelial cells were passaged from the 10 th or 15 th generation to the 40 th or 45 th generation and cultured with or without bavachalcone in DMEM containing $10 \%$ serum. In a part of the experiment, HUVECs cultured with ROR $\alpha$ receptor inhibitor VPR-66 (NBP2-29335, Novus Biologicals, Littleton, CO, USA) were used. For senescence staining, the cellular senescence assay kit (Cell Biolabs, Inc., San Diego, CA, USA) was used. Rat aortal tissue and HUVECs were fixed in $1 \%$ formaldehyde containing $0.2 \%$ glutaraldehyde in PBS for $5 \mathrm{~min}$ at room temperature, rinsed 


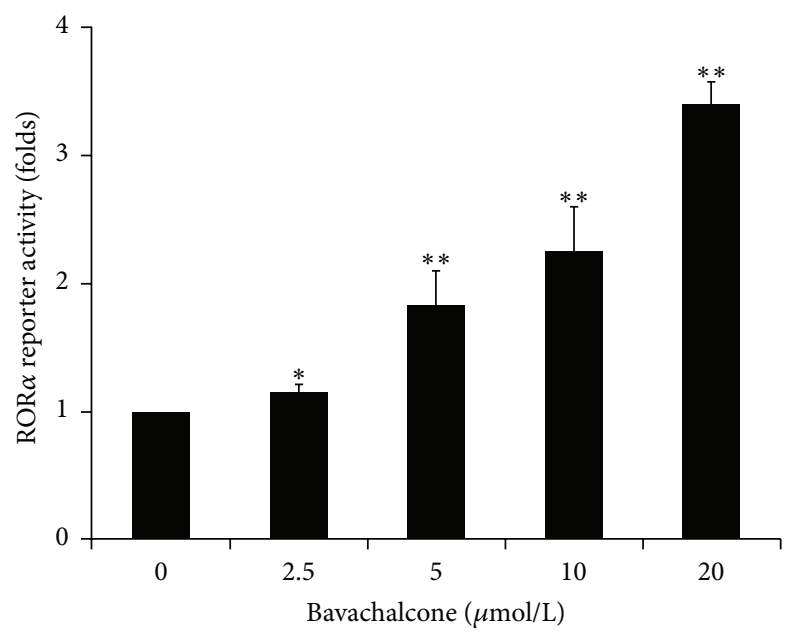

FIGURE 1: Bavachalcone-activated ROR $\alpha$ reporter luciferase activity. Transient transfected HEK293 cells containing ROR $\alpha$ (3× RORE) reporter luciferase plasmids were stimulated with the indicated doses of bavachalcone for $16 \mathrm{~h}$. The cells were lysed and analyzed for luciferase activity $(n=3)$. Data are expressed as the mean \pm SD. ${ }^{*} P<0.05$ versus vehicle control; ${ }^{* *} P<0.01$ versus vehicle control.

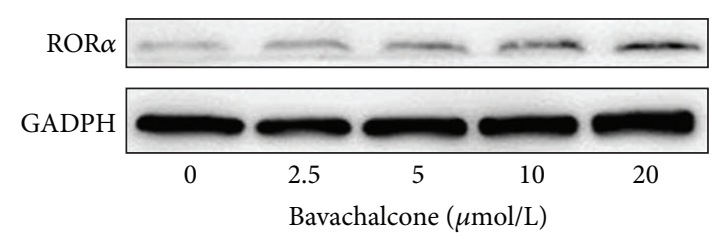

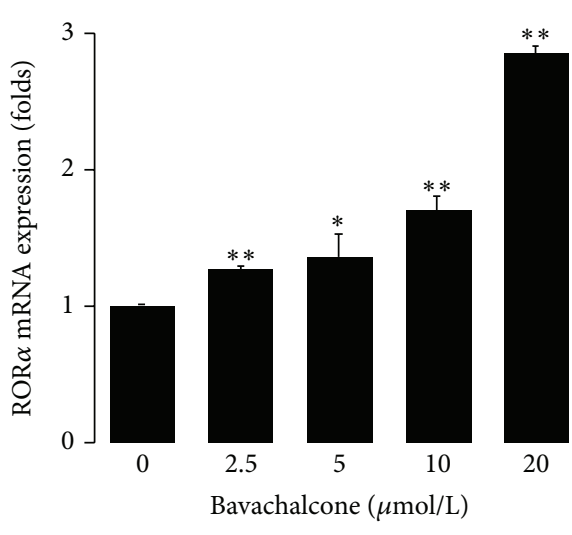

(a)

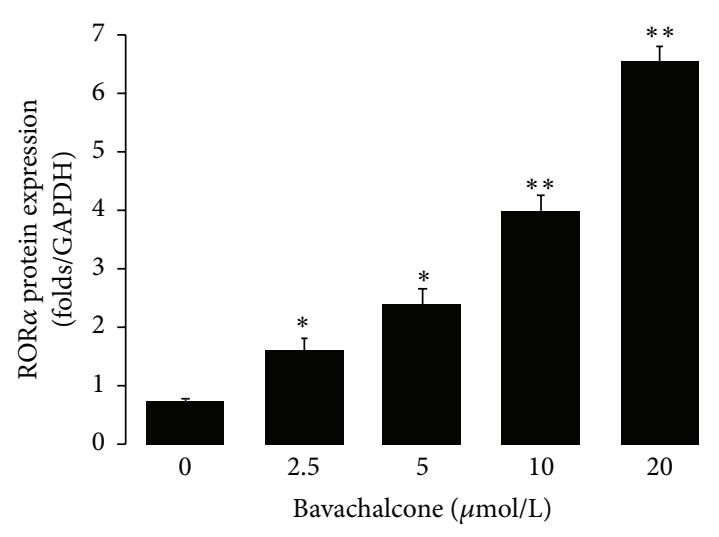

(b)

FIgURE 2: Bavachalcone induced ROR $\alpha$ expression. HUVECs were treated with $20 \mu \mathrm{mol} / \mathrm{L}$ of bavachalcone for $24 \mathrm{~h}$, and mRNA (a) and protein (b) expression levels were measured using real-time quantitative PCR or western blotting $(n=3$ each). Data are expressed as the mean \pm SD. ${ }^{*} P<0.05$ versus vehicle control; ${ }^{* *} P<0.01$ versus vehicle control.

with PBS, and incubated at $37^{\circ} \mathrm{C}$ with a fresh $\beta$-galactosidase stain solution: $1 \mathrm{mg} / \mathrm{mL}$ 5-bromo-4-chloro-3-indolyl $\beta$-D galactoside, $40 \mathrm{mmol} / \mathrm{L}$ citric acid/sodium phosphate $(\mathrm{pH}$ 6.0), $5 \mathrm{mmol} / \mathrm{L}$ potassium ferrocyanide, $150 \mathrm{mmol} / \mathrm{L} \mathrm{NaCl}$, and $2 \mathrm{mmol} / \mathrm{L} \mathrm{MgCl}_{2}$.

2.7. Statistical Analysis. Data are expressed as the mean \pm standard deviation (SD). Paired $t$-test analysis and one-way ANOVA were used to compare the differences between and within the groups. All statistical analyses were performed using SPSS Version 15.0 or GraphPad Prism 5. $P$ value less than 0.05 was considered statistically significant.

\section{Results}

Bavachalcone Induces ROR $\alpha 1$ Expression and Regulates Bmal1 Circadian Rhythm. In the screening of natural ingredient activity, bavachalcone exhibited characteristics that can activate ROR $\alpha 1$ expression (Table 1). Bavachalcone's dose effect relationship showed that it dose-dependently activated ROR $\alpha 1$ luciferase reporter gene activity, causing a more than 3 -fold increase at a dose of $20 \mu \mathrm{mol} / \mathrm{L}(n=3, P<0.01$, Figure 1). Moreover, bavachalcone dose-dependently induced ROR $\alpha 1$ expression in mRNA and protein levels, causing a more than 2-fold increase in mRNA expression $(n=3$, 


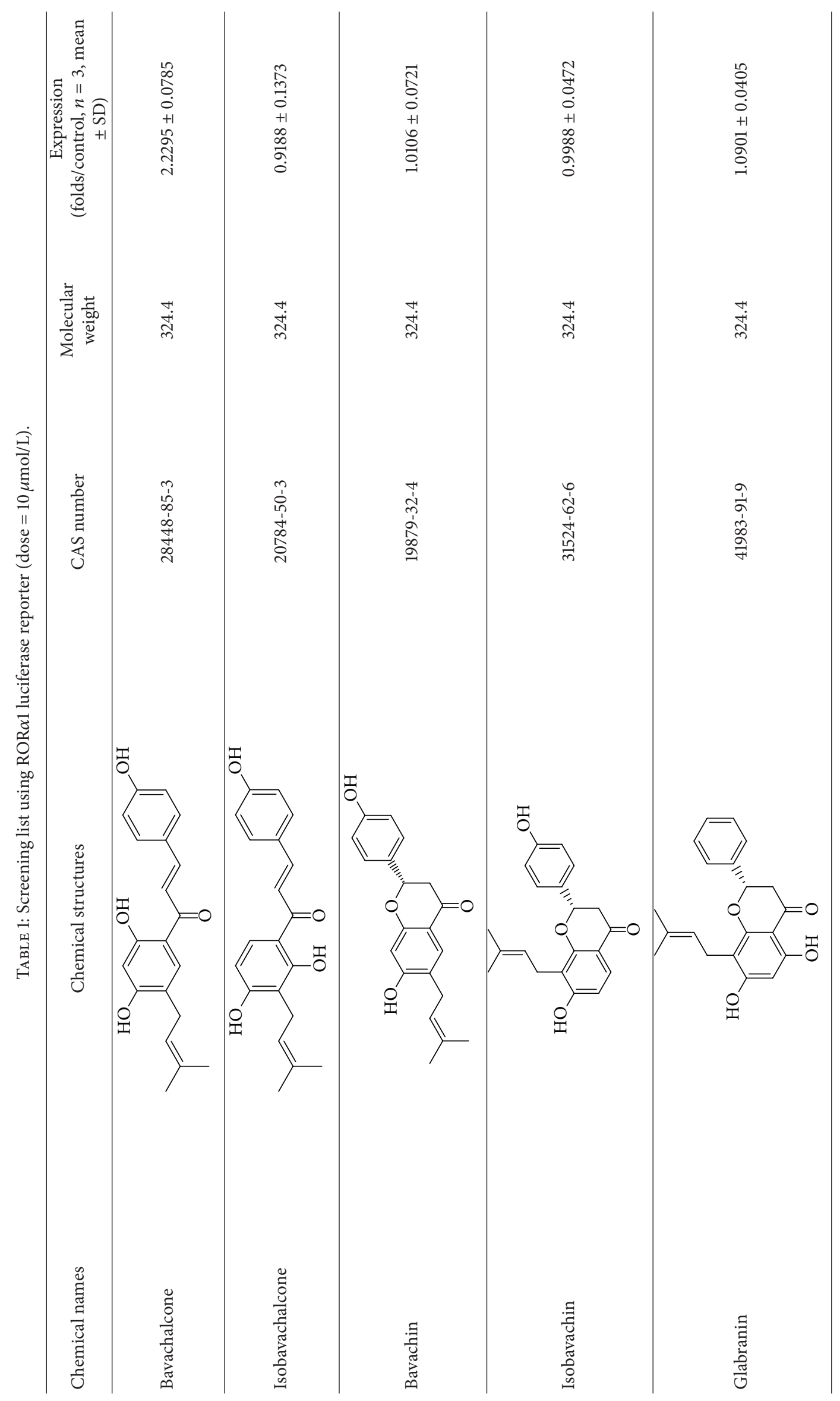




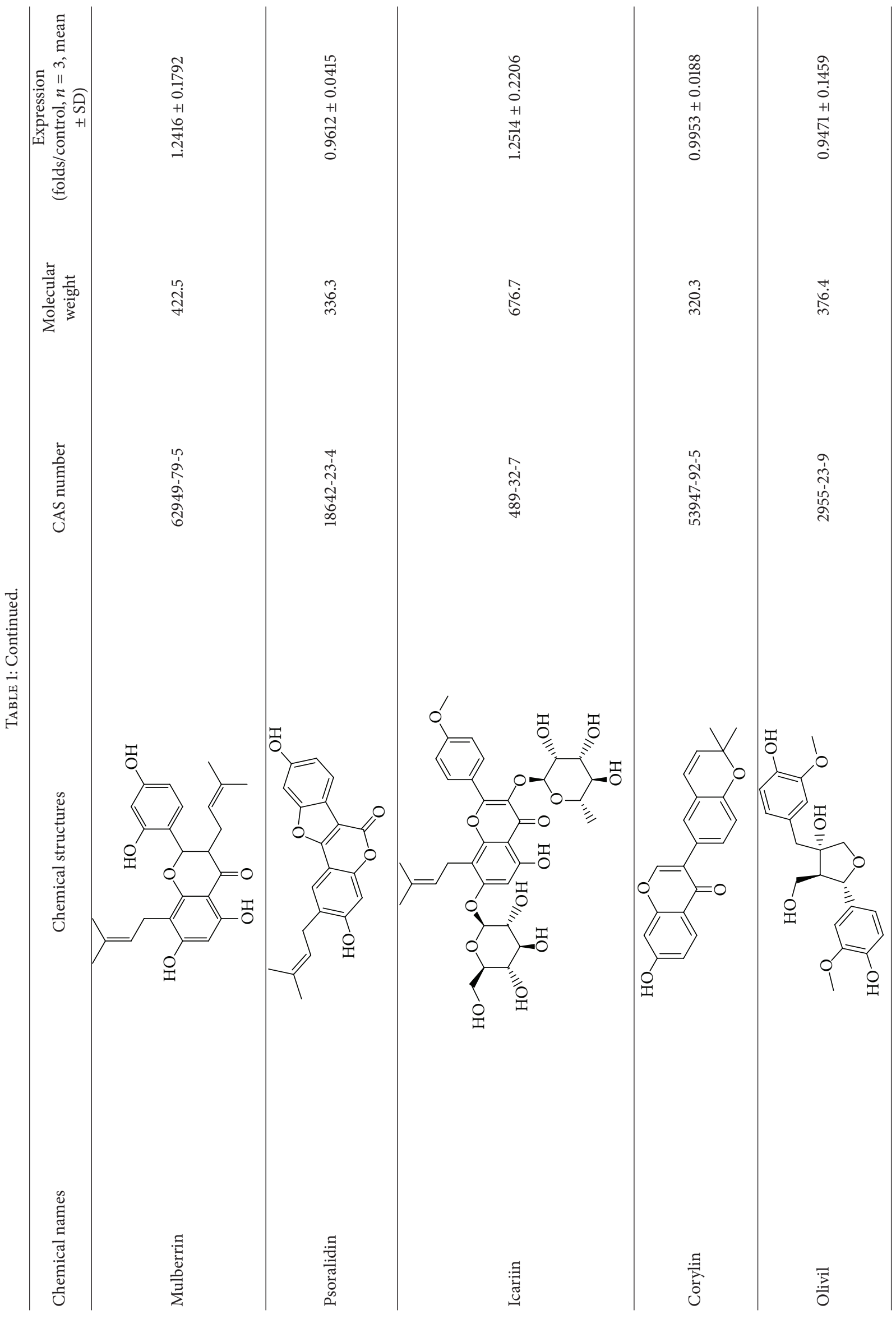




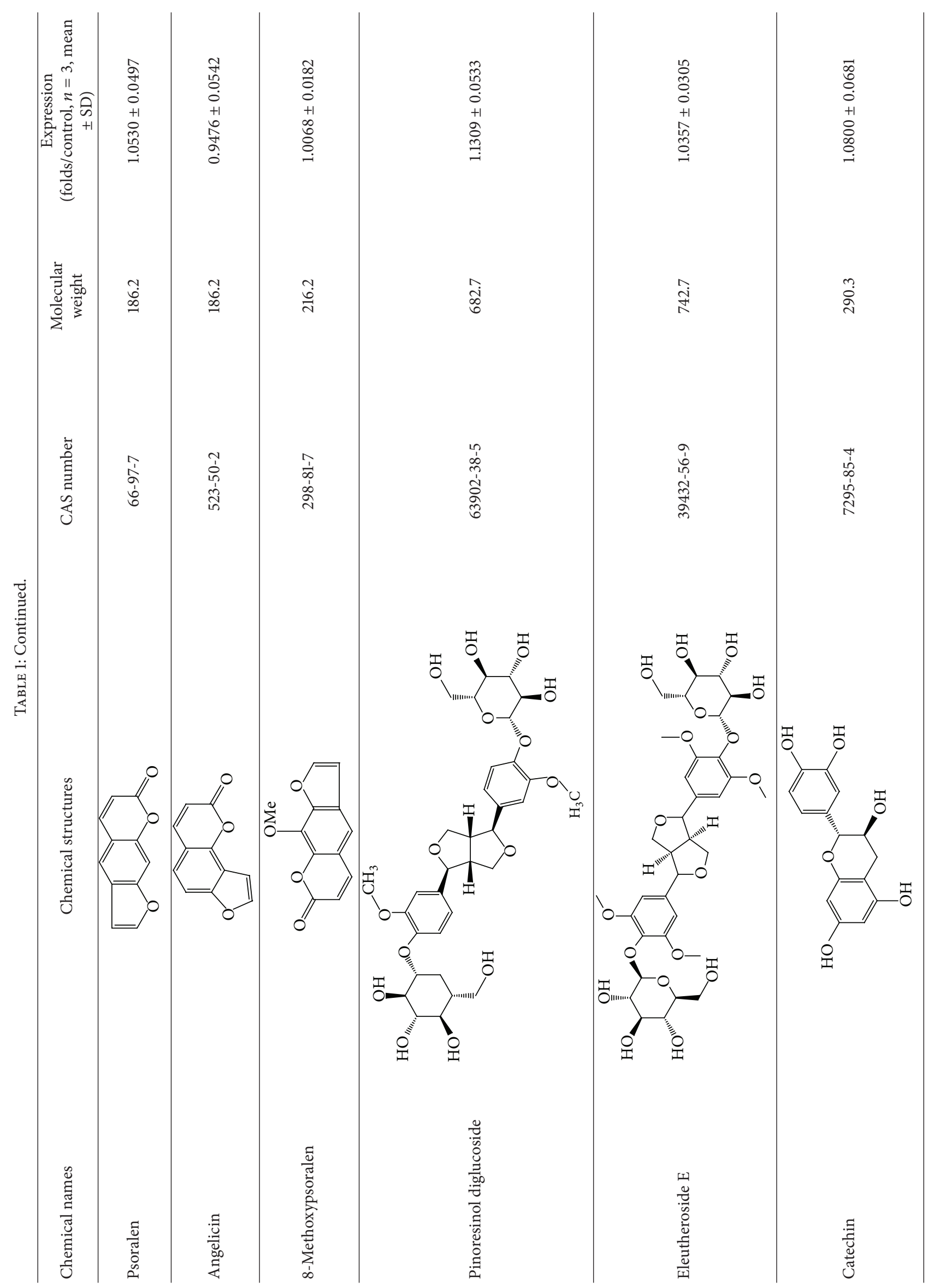




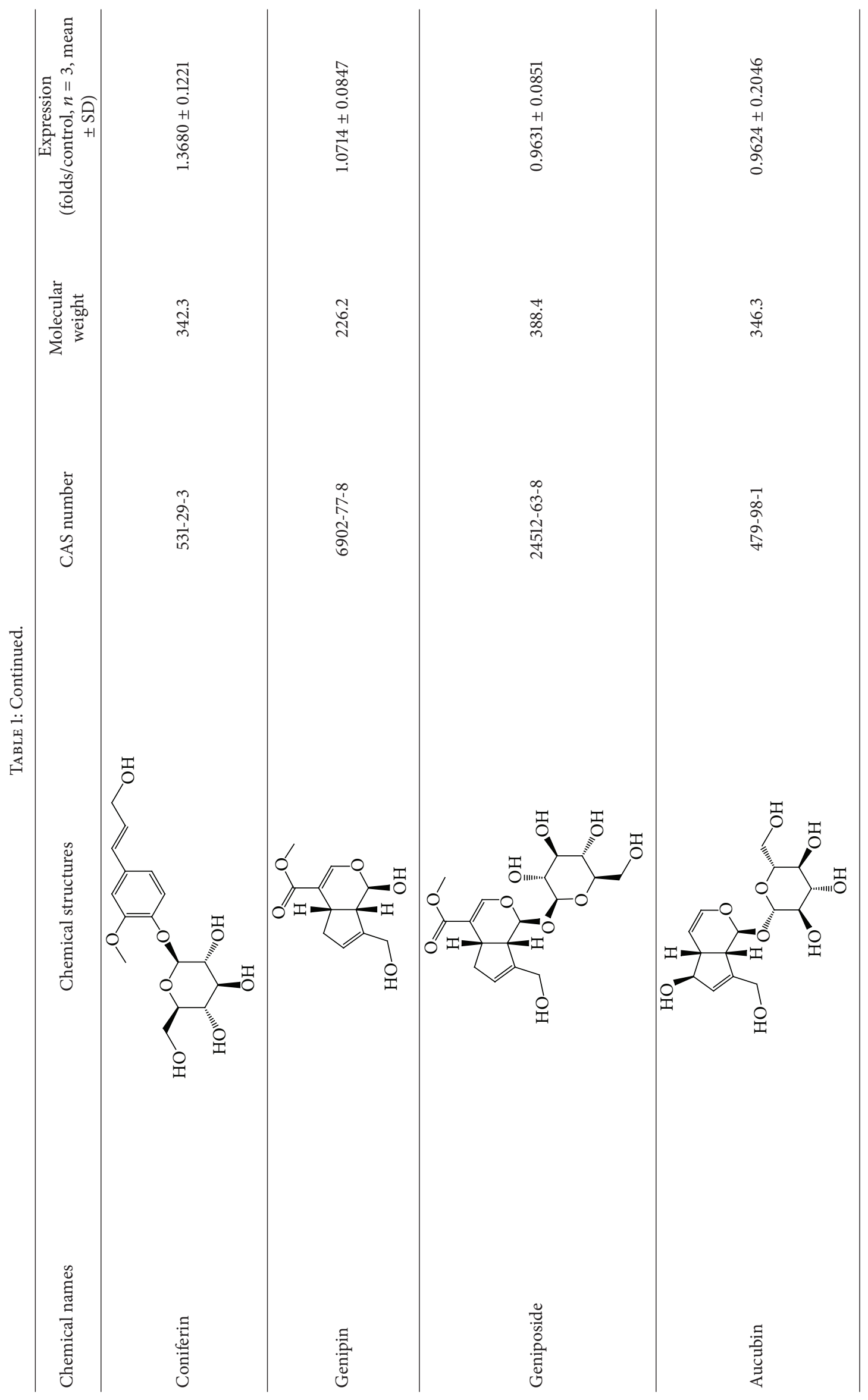




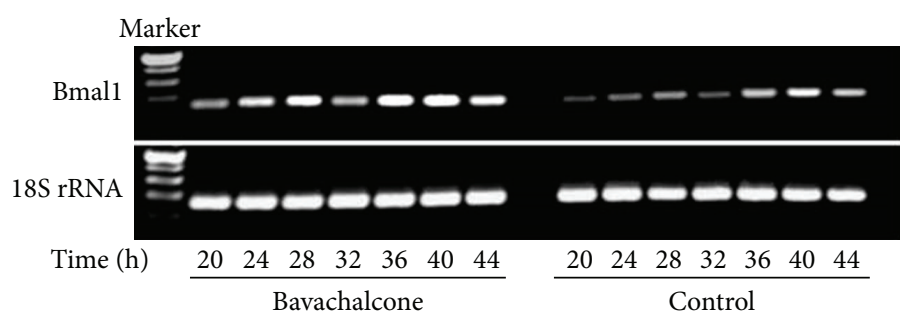

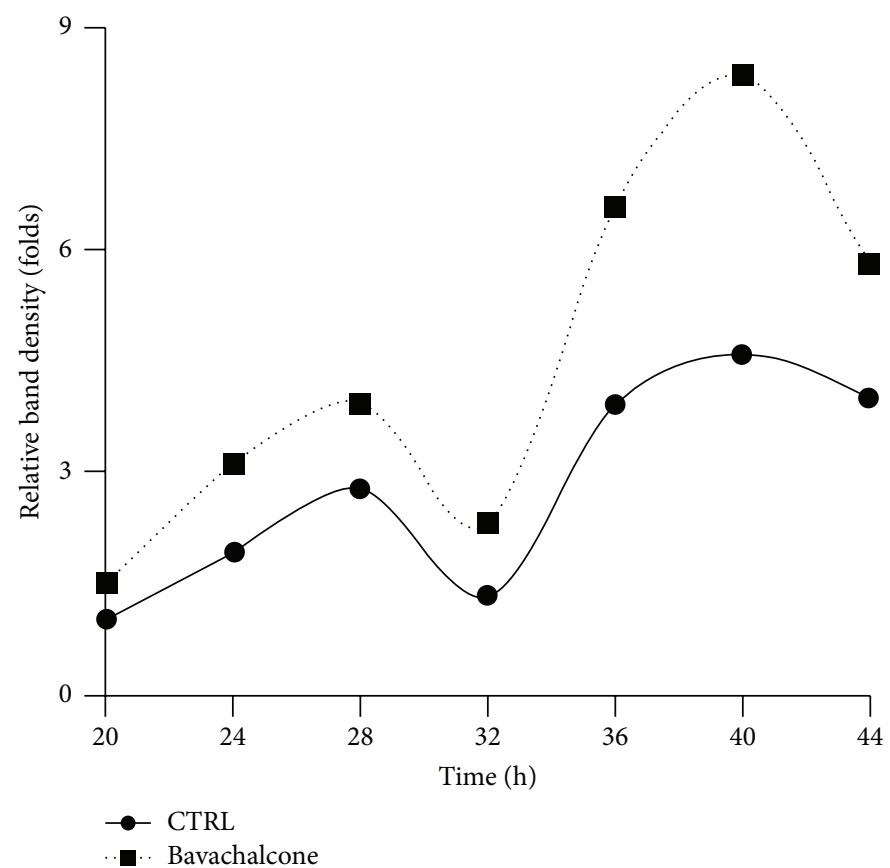

(a)

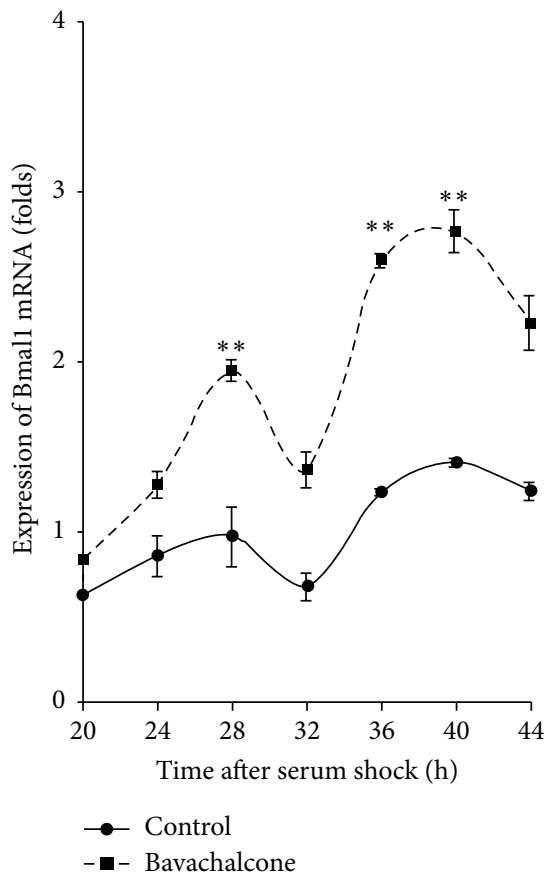

(b)

FIGURE 3: Bavachalcone-enlarged expression amplitude of the circadian gene Bmall. After HUVECs were treated with 50\% serum shock for $1 \mathrm{~h}$, the medium was replaced with $10 \%$ serum DMEM with or without $20 \mu \mathrm{mol} / \mathrm{L}$ bavachalcone. The mRNA expression levels at the indicated time were measured using semiquantitative (a) and real-time quantitative PCR (b) $(n=3$ each). Data are expressed as the mean \pm SD. ${ }^{* *} P<0.01$ versus vehicle control.

$P<0.01$, Figure 2(a)) and a 6-fold increase in protein expression $(n=3, P<0.01$, Figure $2(\mathrm{~b}))$ at a dose of $20 \mu \mathrm{mol} / \mathrm{L}$. Furthermore, bavachalcone dynamically regulated circadian gene Bmall mRNA expression, a ROR $\alpha 1$ downstream signal. As shown in Figures 3(a) and 3(b), from 24 to $48 \mathrm{~h}$ after $50 \%$ serum shock, treatment with bavachalcone enhanced the circadian amplitude of Bmall mRNA expression on 28,36 , and $40 \mathrm{~h}$, which was far wider than other time points compared with the control, with the differences being significant $(n=3, P<0.01)$ at each time point.

Bavachalcone Suppressed Replicative Senescence of Endothelial Cells Partially through ROR $\alpha 1-B m a l 1$ Pathway. Bavachalcone saliently delayed the replicative senescence of endothelial cells; bavachalcone treatment reduced the senescent cell ratio from $26.4 \% \pm 3.0 \%$ to $11.3 \% \pm 1.2 \%$ at the p40 passage ( $n=5, P<0.01$, Figures 4(a) and 4(b)). Subsequently, to determine the relationship between cellular senescence and $\operatorname{ROR} \alpha$, endothelial cells were incubated with the $\operatorname{ROR} \alpha$ inhibitor VPR-66 for $24 \mathrm{~h}$. The results revealed that VPR66 partially antagonized the antiaging effect of bavachalcone $(n=5, P<0.05$, Figures 5(a) and 5(b)). To clearly define the status of cellular senescence, the mRNA expression of $\mathrm{p} 16^{\mathrm{ink} 4 \mathrm{a}}$ and IL- $1 \alpha$ was determined. Vascular endothelial cells undergoing replicative senescence exhibited increased expression of $\mathrm{p} 16^{\text {ink4a }}$ and IL- $1 \alpha$ mRNA, but their expression was significantly suppressed by bavachalcone $(n=3$ each, $P<0.01$, Figures 5(c) and 5(d)). However, coincubation with the ROR $\alpha$ inhibitor VPR-66 for $24 \mathrm{~h}$ partially reversed the inhibitory effect of bavachalcone on the mRNA expressions of $\mathrm{p} 16^{\mathrm{ink} 4 \mathrm{a}}$ and IL- $1 \alpha(n=3, P<0.05$, Figures $5(\mathrm{c})$ and $5(\mathrm{~d}))$.

\section{Discussion}

ROR $\alpha$ is a member of the ROR family, and its ROR $\alpha$ function has been implicated in several pathological processes, such as cancer, autoimmune diseases, inflammation, osteoporosis, and metabolic syndrome [16]. Our results show that 


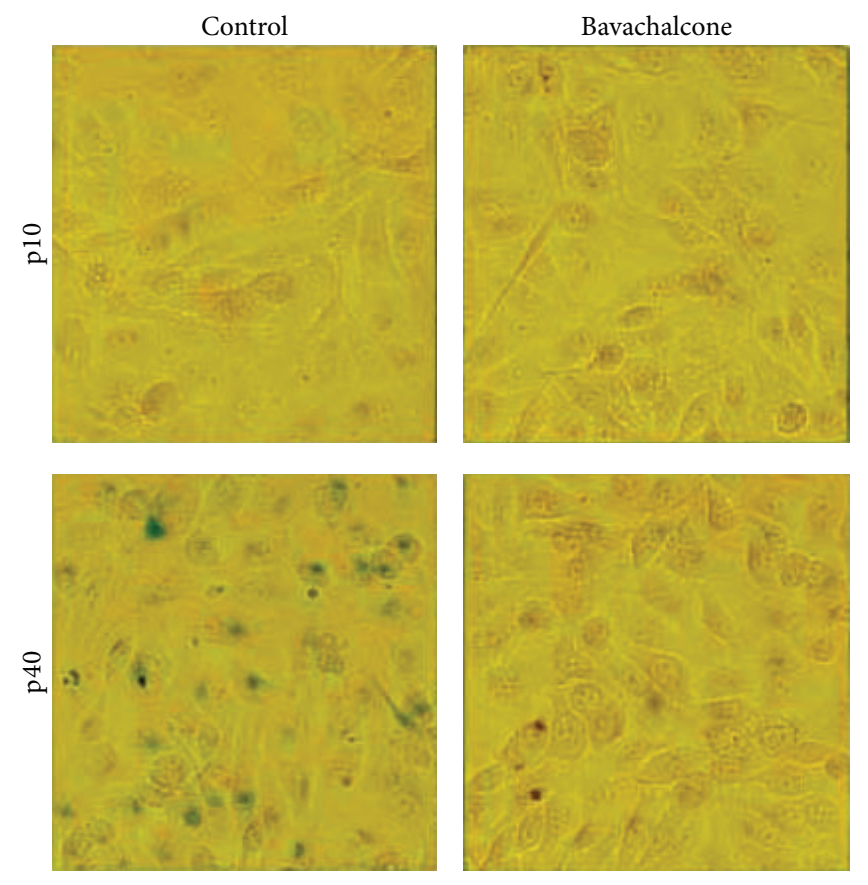

(a)

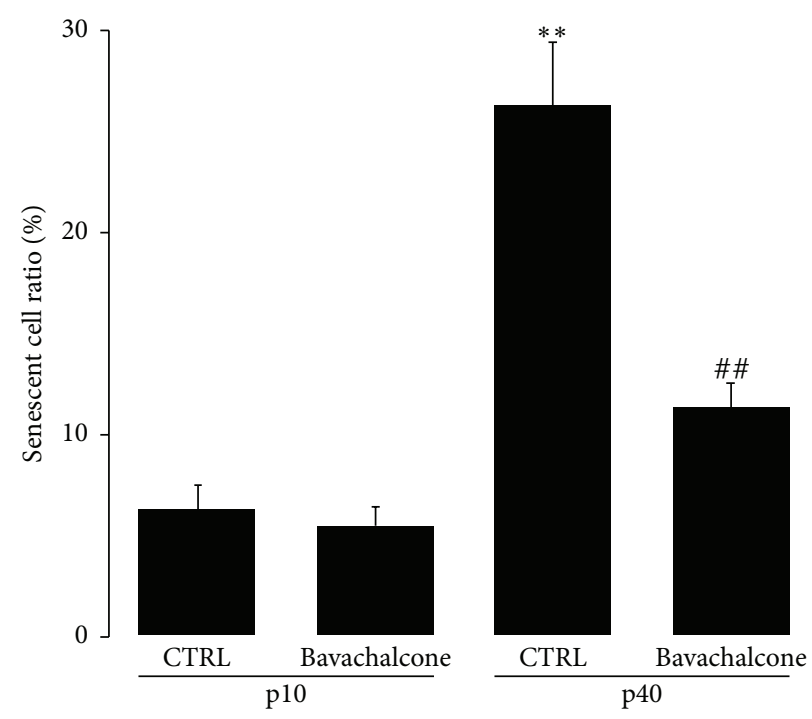

(b)

FIGURE 4: Bavachalcone-delayed senescence of HUVEC. ( $a$ and b) HUVECs were passaged from the 10th to the 40th generation and cultured with or without bavachalcone. Subsequently, the cells were dyed using a $\beta$-galactosidase senescence assay kit. The percentage of senescent cells was obtained by counting more than 500 cells in each sample $\left(n=5\right.$ each). Data are expressed as the mean \pm SD. ${ }^{* *} P<0.01$ versus p10 passage vehicle control. ${ }^{\# \#} P<0.01$ versus p40 passage vehicle control.

bavachalcone activates ROR $\alpha 1$ luciferase reporter activity and mRNA and protein expression. Our previous findings show that bavachalcone activates AMPK activity and induces MnSOD expression [14]. In addition, ROR $\alpha 1$ activates AMPK activity $[17,18]$, and reduces oxidative stress through the induction of MnSOD and GPxl expression [9]; our results are consistent with these reports. Numerous studies have indicated that the transcriptional activating function of $\operatorname{ROR} \alpha 1$ is regulated not only by its natural ligands, such as cholesterol and cholesterol derivatives, but also by synthetic ligands [16].

Our results show that bavachalcone delays cell senescence and inhibits mRNA expression of p16 ${ }^{\text {ink4a }}$ (a maker of replicative senescence [19]) and IL-1 $\alpha$ (a proinflammatory cytokine of the senescence-associated secretory phenotype [20]). These inhibitory effects could be partially reversed by the ROR $\alpha$ inhibitor VPR-66. The Bmall promoter region contains ROR response elements where the ROR families can bind [21], supporting our results that the dynamic expression of Bmall gene is regulated by bavachalcone-enhanced ROR $\alpha 1$ activity. Studies have reported that deficiency of Bmall increases vascular superoxide production; endothelial nitric oxide synthase uncoupling; and COX-2, Nox4, MMP2, and MMP9 expressions and underlies vascular stiffness [2224]. In addition, studies have shown that deficiency of the circadian clock transcriptional factor BMAL1 impairs glucose homeostasis, exhibits premature age-associated disorders, reduces lifespan [25-27], and increases sensitivity to genotoxic stress [28]. A recent study reported that reactive oxygen species exhibits a wave in the circadian rhythm in the brains of wild-type mice, but not in Bmall deficient mice [29]. In addition, deficiency of clock gene, a Bmall partner, reduces lifespan and increases age-related characteristics [30].

In conclusion, through a luciferase assay, we showed that bavachalcone induced $\mathrm{ROR} \alpha 1$ expressions at luciferase reporter, mRNA, and protein levels in human endothelial cells and dynamically regulated Bmall mRNA expression. In addition, bavachalcone suppressed replicative senescence in human endothelial cells partially through the $\operatorname{ROR} \alpha$ Bmall pathway. Our results demonstrate that bavachalcone, a natural medicine ingredient, has a pharmacological function in regulating $\mathrm{ROR} \alpha$.

\section{Conflict of Interests}

The authors declare no conflict of interests.

\section{Acknowledgments}

This work was supported by grants from the Specialized Research Fund for the National Natural Science Foundation 

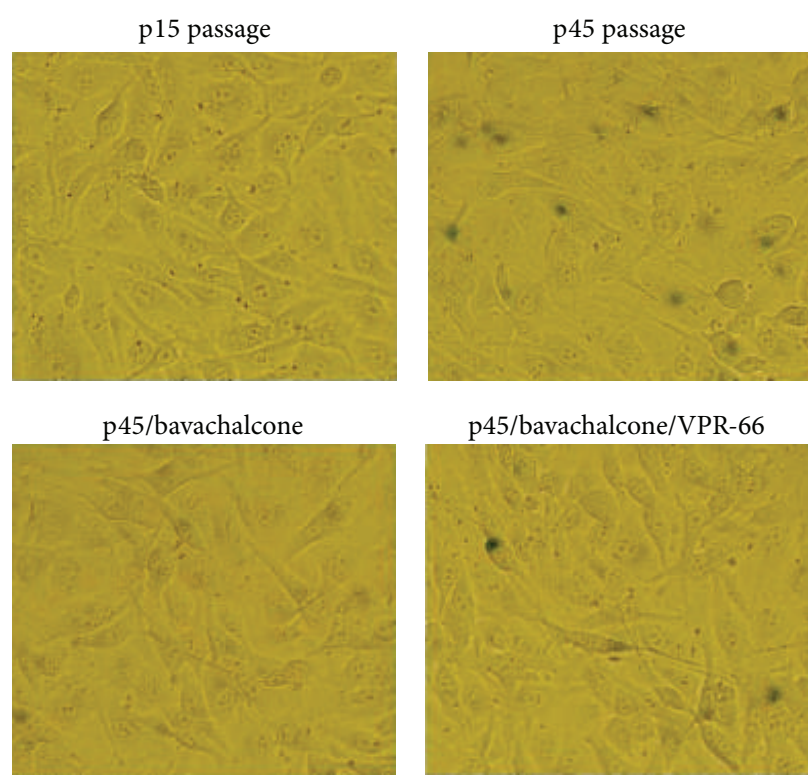

p45/bavachalcone/VPR-66

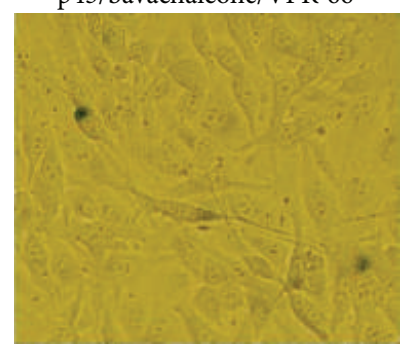

(a)

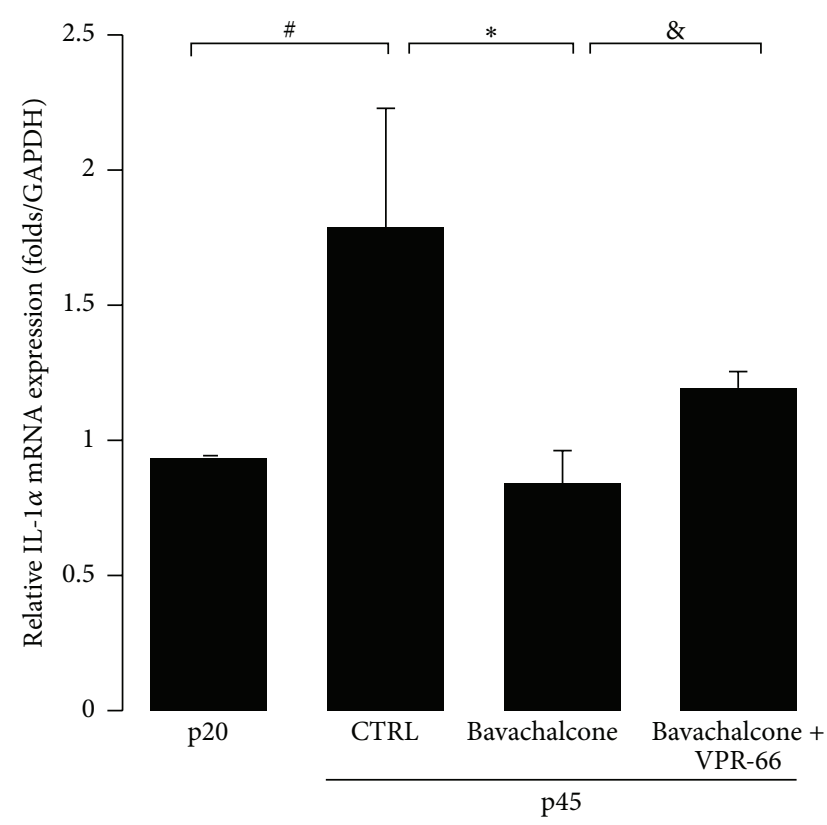

(c)

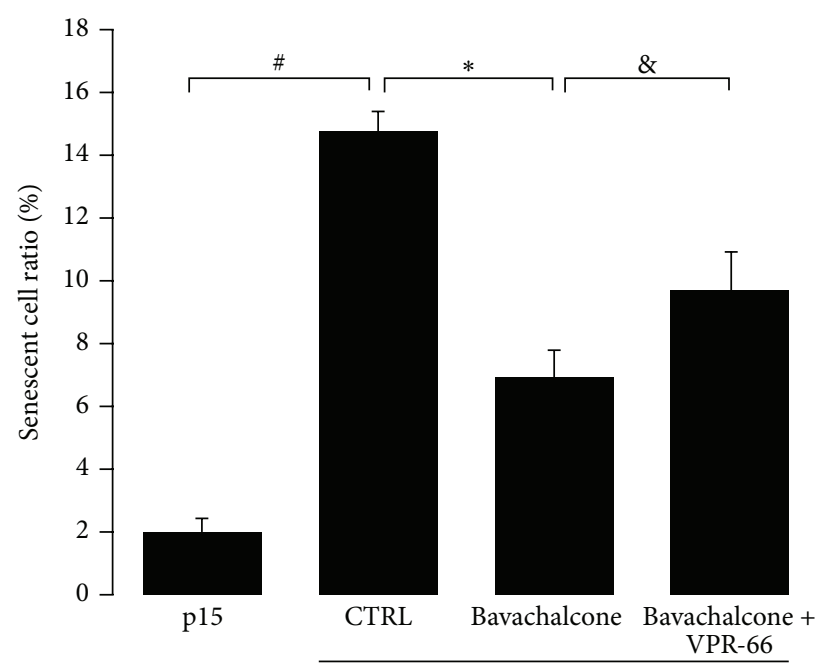

(b)

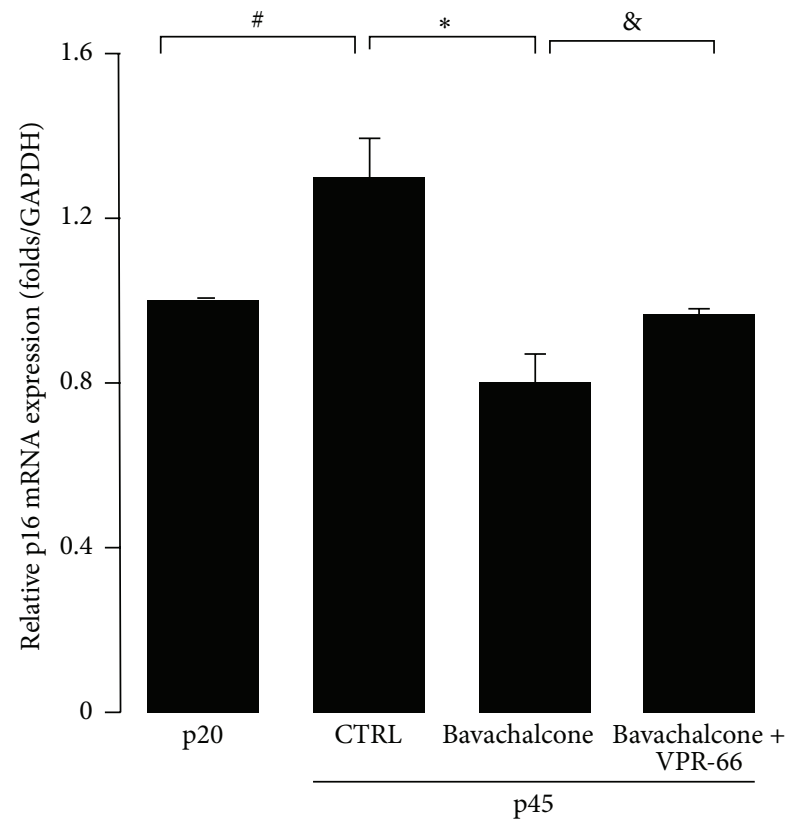

(d)

FIGURE 5: Bavachalcone-delayed cellular senescence, partially through ROR $\alpha$-Bmall pathway. ( $\mathrm{a}$ and $\mathrm{b}$ ) HUVECs were passaged from the 15th to the 45th generation and treated with or without bavachalcone and with or without VPR-66, a ROR $\alpha$ inhibitor. Subsequently, the cells were dyed using a $\beta$-galactosidase senescence assay kit. The percentage of senescent cells was obtained by counting more than 500 cells in each sample ( $n=5$ each). (c and d) HUVECs were passaged from the 20th to the 45th generation and treated with or without bavachalcone and with or without VPR-66. The mRNA expression levels were measured using real-time quantitative PCR ( $n=3$ each). Data are expressed as the mean \pm SD. ${ }^{\#} P<0.01$ versus p15 or p20 passages vehicle control; ${ }^{*} P<0.01$ versus p45 passage vehicle control; ${ }^{8} P<0.05$ versus p45 passage bavachalcone treatment.

of China (81274130), the National Natural Science Foundation of China-Youth Fund (81102532), the Doctoral Program of Higher Education of China (20113107110006), the Innovation Program of the Shanghai Municipal Education Commission of China (12ZZ122), the Shanghai 085
Project of Higher Education Connotation Construction (085ZY1202), and the Development Program of Key Disciplines of Traditional Chinese Medicine of the Shanghai Municipal Education Commission of China (ZYX-CXYJ005). 


\section{References}

[1] G. K. Paschos and G. A. FitzGerald, "Circadian clocks and vascular function," Circulation Research, vol. 106, no. 5, pp. 833841, 2010.

[2] S. Besnard, C. Heymes, R. Merval et al., "Expression and regulation of the nuclear receptor $\operatorname{ROR} \alpha$ in human vascular cells," FEBS Letters, vol. 511, no. 1-3, pp. 36-40, 2002.

[3] M. Akashi and T. Takumi, "The orphan nuclear receptor ROR $\alpha$ regulates circadian transcription of the mammalian core-clock Bmall," Nature Structural and Molecular Biology, vol. 12, no. 5, pp. 441-448, 2005.

[4] F. Guillaumond, H. Dardente, V. Giguère, and N. Cermakian, "Differential control of Bmall circadian transcription by REVERB and ROR nuclear receptors," Journal of Biological Rhythms, vol. 20, no. 5, pp. 391-403, 2005.

[5] A. Mamontova, S. Séguret-Macé, B. Esposito et al., "Severe atherosclerosis and hypoalphalipoproteinemia in the staggerer mouse, a mutant of the nuclear receptor ROR $\alpha$," Circulation, vol. 98, no. 24, pp. 2738-2743, 1998.

[6] A. Genoux, H. Dehondt, A. Helleboid-Chapman et al., "Transcriptional regulation of apolipoprotein A5 gene expression by the nuclear receptor ROR $\alpha$," Arteriosclerosis, Thrombosis, and Vascular Biology, vol. 25, no. 6, pp. 1186-1192, 2005.

[7] E. Raspé, H. Duez, P. Gervois et al., "Transcriptional regulation of apolipoprotein C-III gene expression by the orphan nuclear receptor ROR $\alpha$," The Journal of Biological Chemistry, vol. 276, no. 4, pp. 2865-2871, 2001.

[8] H. Migita, N. Satozawa, J.-H. Lin, J. Morser, and K. Kawai, "ROR $\alpha 1$ and ROR $\alpha 4$ suppress TNF- $\alpha$-induced VCAM- 1 and ICAM-1 expression in human endothelial cells," FEBS Letters, vol. 557, no. 1-3, pp. 269-274, 2004.

[9] Y. Han, H. Kim, E. Kim et al., "ROR $\alpha$ decreases oxidative stress through the induction of SOD2 and GPx1 expression and thereby protects against nonalcoholic steatohepatitis in mice," Antioxidants \& Redox Signaling, vol. 21, no. 15, pp. 2083-2094, 2014.

[10] M. Miyazaki, E. Schroder, S. E. Edelmann et al., "Age-associated disruption of molecular clock expression in skeletal muscle of the spontaneously hypertensive rat," PLoS ONE, vol. 6, no. 11, Article ID e27168, 2011.

[11] M. H. Lee, J. Y. Kim, and J.-H. Ryu, "Prenylflavones from Psoralea corylifolia inhibit nitric oxide synthase expression through the inhibition of $\mathrm{I}-\kappa \mathrm{B}-\alpha$ degradation in activated microglial cells," Biological and Pharmaceutical Bulletin, vol. 28, no. 12, pp. 2253-2257, 2005.

[12] C. K. Park, Y. Lee, E.-J. Chang et al., "Bavachalcone inhibits osteoclast differentiation through suppression of NFATc1 induction by RANKL," Biochemical Pharmacology, vol. 75, no. 11, pp. 2175-2182, 2008.

[13] L. Shan, S. Yang, G. Zhang et al., "Comparison of the inhibitory potential of bavachalcone and corylin against UDPglucuronosyltransferases," Evidence-Based Complementary and Alternative Medicine, vol. 2014, Article ID 958937, 6 pages, 2014.

[14] Y. Dang, S. Ling, J. Duan, J. Ma, R. Ni, and J.-W. Xu, "Bavachalcone-induced manganese superoxide dismutase expression through the AMP-activated protein kinase pathway in human endothelial cells," Pharmacology, vol. 95, no. 3-4, pp. 105-110, 2015.

[15] T. Hayashi, H. Matsui-Hirai, A. Miyazaki-Akita et al., "Endothelial cellular senescence is inhibited by nitric oxide: implications in atherosclerosis associated with menopause and diabetes,"
Proceedings of the National Academy of Sciences of the United States of America, vol. 103, no. 45, pp. 17018-17023, 2006.

[16] L. A. Solt and T. P. Burris, "Action of RORs and their ligands in (patho)physiology," Trends in Endocrinology \& Metabolism, vol. 23, no. 12, pp. 619-627, 2012.

[17] E.-J. Kim, Y.-S. Yoon, S. Hong et al., "Retinoic acid receptorrelated orphan receptor $\alpha$-induced activation of adenosine monophosphate-activated protein kinase results in attenuation of hepatic steatosis," Hepatology, vol. 55, no. 5, pp. 1379-1388, 2012.

[18] E. J. Kim, Y. K. Choi, Y. H. Han, H. J. Kim, I. K. Lee, and M. O. Lee, "ROR $\alpha$ suppresses proliferation of vascular smooth muscle cells through activation of AMP-activated protein kinase," International Journal of Cardiology, vol. 175, no. 3, pp. 515-521, 2014.

[19] D. A. Alcorta, Y. Xiong, D. Phelps, G. Hannon, D. Beach, and J. C. Barrett, "Involvement of the cyclin-dependent kinase inhibitor p16 (INK4a) in replicative senescence of normal human fibroblasts," Proceedings of the National Academy of Sciences of the United States of America, vol. 93, no. 24, pp. 13742-13747, 1996.

[20] A. V. Orjalo, D. Bhaumik, B. K. Gengler, G. K. Scott, and J. Campisi, "Cell surface-bound IL-1alpha is an upstream regulator of the senescence-associated IL-6/IL-8 cytokine network," Proceedings of the National Academy of Sciences of the United States of America, vol. 106, no. 40, pp. 17031-17036, 2009.

[21] M. Akashi and T. Takumi, "The orphan nuclear receptor ROR $\alpha$ regulates circadian transcription of the mammalian core-clock Bmall," Nature Structural and Molecular Biology, vol. 12, no. 5, pp. 441-448, 2005.

[22] C. B. Anea, M. Irfan Ali, J. M. Osmond et al., "Matrix metalloproteinase 2 and 9 dysfunction underlie vascular stiffness in circadian clock mutant mice," Arteriosclerosis, Thrombosis, and Vascular Biology, vol. 30, no. 12, pp. 2535-2543, 2010.

[23] C. B. Anea, B. Cheng, S. Sharma et al., "Increased superoxide and endothelial NO synthase uncoupling in blood vessels of Bmall-knockout mice," Circulation Research, vol. 111, no. 9, pp. 1157-1165, 2012.

[24] C. B. Anea, M. Zhang, F. Chen et al., "Circadian clock control of Nox 4 and reactive oxygen species in the vasculature," PLoS ONE, vol. 8, no. 10, Article ID e78626, 2013.

[25] R. V. Kondratov, A. A. Kondratova, V. Y. Gorbacheva, O. V. Vykhovanets, and M. P. Antoch, "Early aging and age-related pathologies in mice deficient in BMAL1, the core componentof the circadian clock," Genes \& Development, vol. 20, pp. 18681873, 2006.

[26] R. V. Khapre, A. A. Kondratova, O. Susova, and R. V. Kondratov, "Circadian clock protein BMAL1 regulates cellular senescence in vivo," Cell Cycle, vol. 10, no. 23, pp. 4162-4169, 2011.

[27] R. V. Khapre, A. A. Kondratova, S. Patel et al., "BMAL1dependent regulation of the mTOR signaling pathway delays aging," Aging, vol. 6, no. 1, pp. 48-57, 2014.

[28] M. P. Antoch and R. V. Kondratov, "Circadian proteins and genotoxic stress response," Circulation Research, vol. 106, no. 1, pp. 68-78, 2010.

[29] T. A. Wang, Y. V. Yu, G. Govindaiah et al., "Circadian rhythm of redox state regulates excitability in suprachiasmatic nucleus neurons," Science, vol. 337, no. 6096, pp. 839-842, 2012.

[30] Y. V. Dubrovsky, W. E. Samsa, and R. V. Kondratov, "Deficiency of circadian protein CLOCK reduces lifespan and increases agerelated cataract development in mice," Aging, vol. 2, no. 12, pp. 936-944, 2010. 


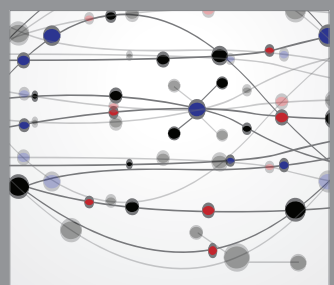

The Scientific World Journal
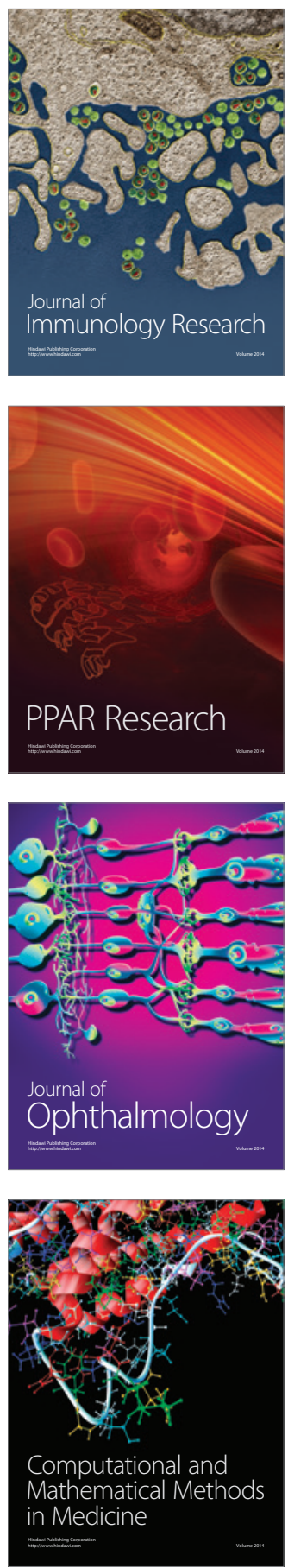

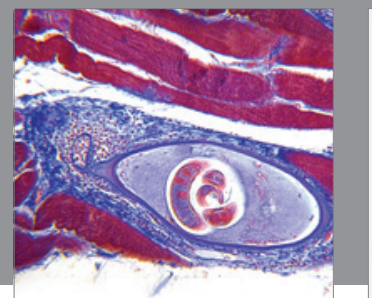

Gastroenterology

Research and Practice
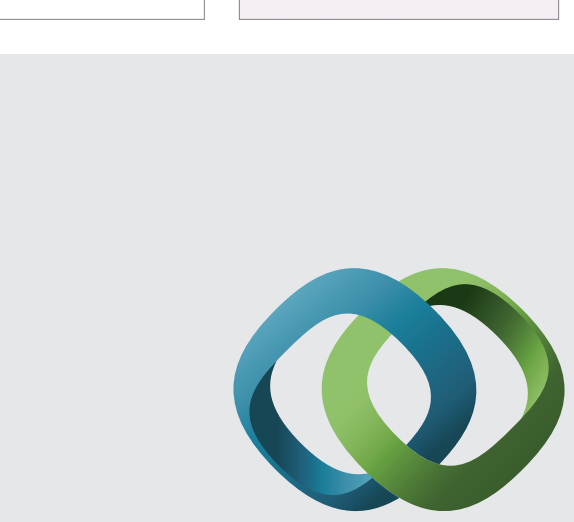

\section{Hindawi}

Submit your manuscripts at

http://www.hindawi.com
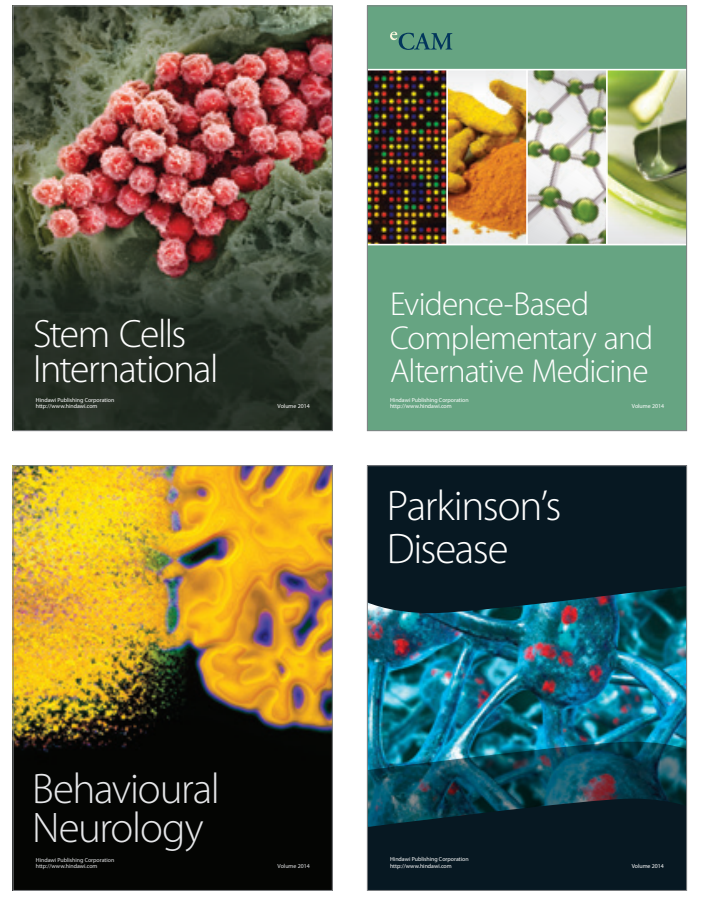
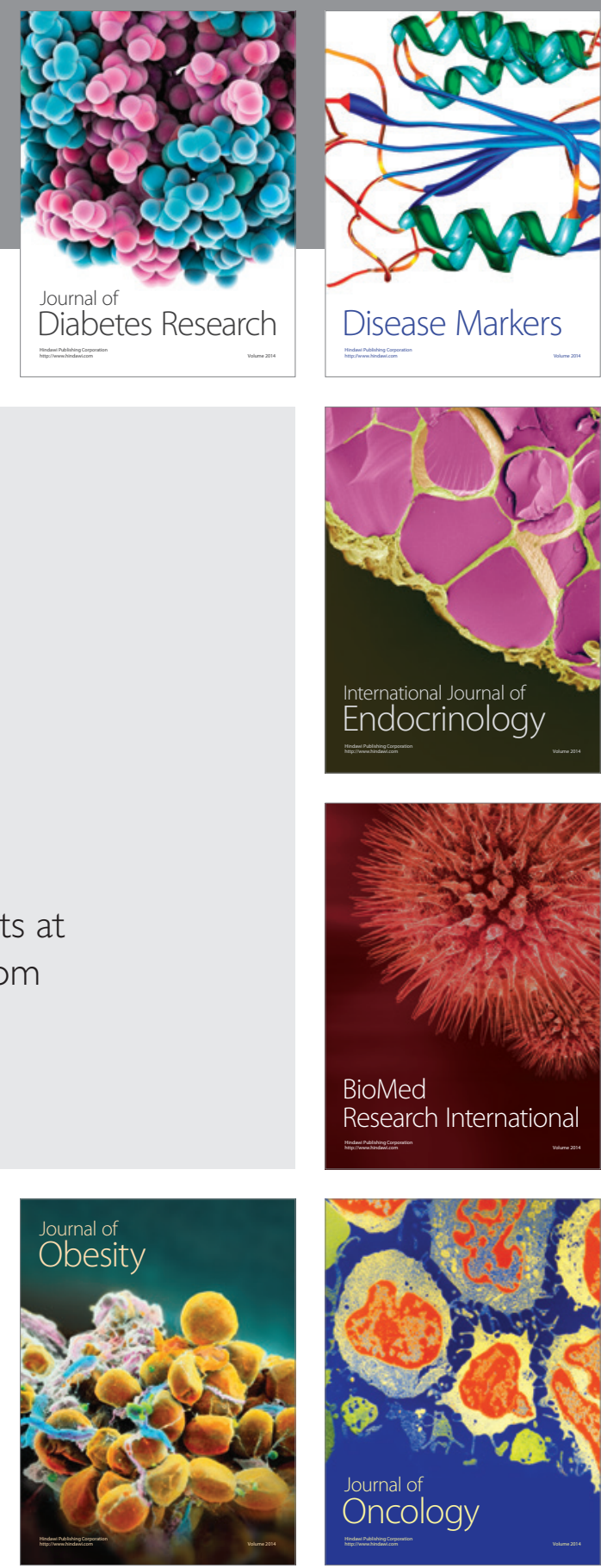

Disease Markers
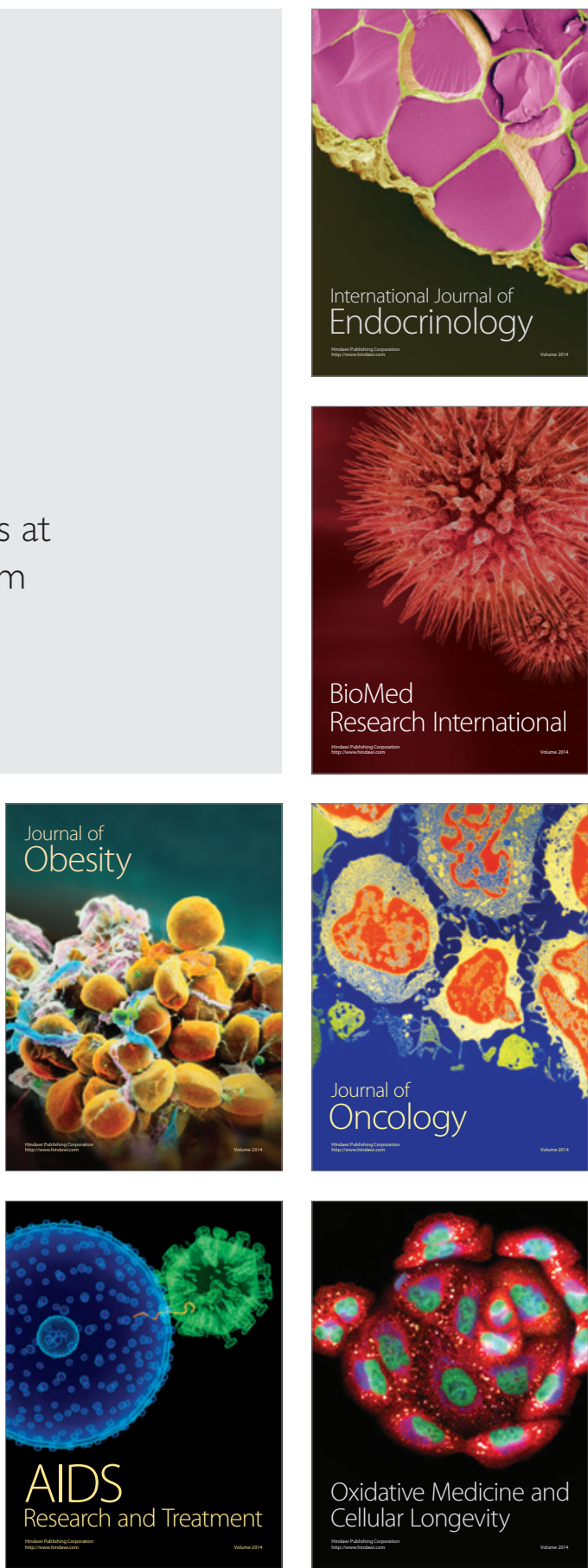